\title{
Unconventional spin currents in magnetic films
}

\author{
Dmytro A. Bozhko $\odot,{ }^{1,2, *}$ Halyna Yu. Musiienko-Shmarova $\odot,{ }^{1, \dagger}$ Vasyl S. Tiberkevich,, , Andrei N. Slavin $\odot,{ }^{3, \S}$

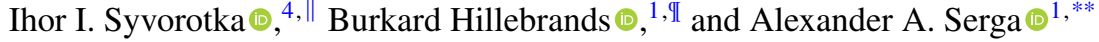 \\ ${ }^{1}$ Fachbereich Physik and Landesforschungszentrum OPTIMAS, Technische Universität Kaiserslautern, 67663 Kaiserslautern, Germany \\ ${ }^{2}$ Department of Physics and Energy Science, University of Colorado at Colorado Springs, Colorado Springs, Colorado 80918, USA \\ ${ }^{3}$ Department of Physics, Oakland University, Rochester, Michigan 48309, USA \\ ${ }^{4}$ Department of Crystal Physics and Technology, SRC “Carat," 79031 Lviv, Ukraine
}

(Received 7 May 2019; revised manuscript received 26 March 2020; accepted 29 April 2020;

published 12 June 2020)

\begin{abstract}
A spin current-a flow of spin angular momentum — can be carried either by spin-polarized free electrons or by magnons, the quanta of a moving collective oscillation of localized electron spins, i.e., a spin wave. Traditionally, it was assumed that a spin wave in a magnetic film with spin-sink-free surfaces can transfer energy and angular momentum only along its propagation direction. In this work, using Brillouin light-scattering spectroscopy in combination with a theory of dipole-exchange spin-wave spectra, we show that in obliquely magnetized free magnetic films, the in-plane propagation of spin waves is accompanied by a transverse spin current along the film normal without any corresponding transverse transport of energy.
\end{abstract}

DOI: 10.1103/PhysRevResearch.2.023324

\section{INTRODUCTION}

Spin waves (or their quanta, magnons) can be used as carriers of a spin current in spintronic [1,2] and magnonic [3-5] signal-processing devices, as they enable transport of energy and spin angular momentum over long distances along their propagation direction [6-8]. This has been used for concepts in view of novel spintronic data-processing applications, such as planar spin-wave conduits $[9,10]$, magnonic crystals [11,12], logic elements, such as spin-wave majority gates [13], and nonlinear spin-wave devices, such as magnon transistors [14]. Many of these novel elements operate in the dipole-exchange region of a spin-wave spectrum, where the strength of magnetic dipole-dipole and local electrostatic exchange interactions between electron spins is comparable. Most experimental studies of dipole-exchange spin waves have been performed using in-plane magnetized films, where the spin-wave propagation in the long-wavelength part of the spectrum is highly anisotropic due to the influence of the dipolar interaction, and exotic phenomena such as spinwave caustics [15] may appear. Furthermore, the interplay between dipolar and exchange interactions in this magneti-

\footnotetext{
*dbozhko@uccs.edu

†musiien@rhrk.uni-kl.de

*tyberkev@oakland.edu

§slavin@oakland.edu

"igor@ carat.lviv.ua

"hilleb@physik.uni-kl.de

**serga@physik.uni-kl.de
}

zation geometry creates prerequisites for fundamental phenomena such as magnon Bose-Einstein condensation [16-18], magnonic supercurrents [19-21], formation of hybrid magnetoelastic bosons [22], spin superfluidity [23-25], and more. In spin-sink-free in-plane magnetized magnetic films, the energy and spin angular momentum are transported by spin-wave modes along the film plane, and the transverse (along the film normal) profiles of the spin-wave modes are standing waves, which do not exhibit a net transport of energy or spin angular momentum.

However, if a spin sink, i.e., an overlayer with strong spin-orbit coupling (such as $\mathrm{Pt}$ or $\mathrm{Ta}$ ), is attached to the film, the absorption of magnons by such a spin sink leads to the appearance of spin and energy flows along the film normal [26,27]. It allows for the detection of propagating spin waves in a large range of wavelengths [27], and thus it plays an important role in the development of magnon spintronics toward utilization of short-wavelength dipolar-exchange and exchange spin waves in nanosized devices [1].

Here we report that, in contrast with the case of in-plane magnetization, in obliquely magnetized films the transverse profiles of in-plane-propagating dipole-exchange spin waves are only quasistanding, and they may allow for the transport of a spin angular momentum along the film normal even in the absence of any spin sink. It is noteworthy that in the case of no spin absorption at the film boundaries, such currents abstain from a net transport of energy. These unconventional spin currents may be crucially important for applications in modern spintronics [28], where they can substantially influence the effects of spin pumping and spin-transfer torque at a film interface.

\section{GEDANKEN EXPERIMENT}

Creative Commons Attribution 4.0 International license. Further distribution of this work must maintain attribution to the author(s) and the published article's title, journal citation, and DOI.
First we consider a gedanken experiment, such as the one schematically shown in Fig. 1(b), where a spin wave travels 

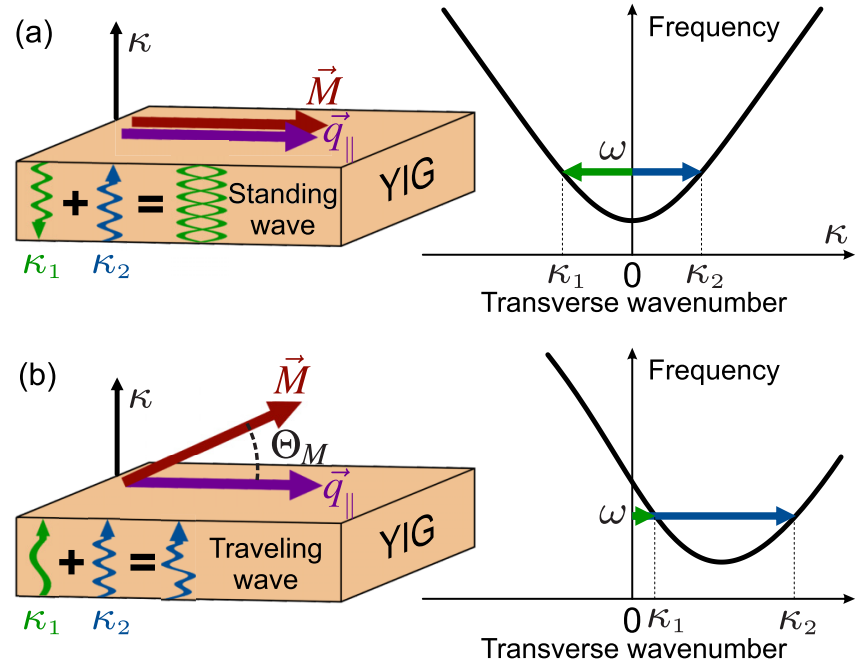

FIG. 1. Standing- and traveling-mode profiles. Dependence of the spin-wave frequency on the transverse (to the direction of wave propagation and along the film normal) wave number $\kappa$ in a ferromagnetic film for different angles $\Theta_{M}$ between the static magnetization and the direction of the wave propagation $\boldsymbol{q}_{\|}$: (a) $\Theta_{M}=0^{\circ}$; (b) $\Theta_{M}>0^{\circ}$.

in-plane in the $+\boldsymbol{q}$ direction. Such a scenario can be realized by using an antenna structure to generate the spin waves, positioned left to the spot, where we detect the spin waves in a Brillouin light-scattering experiment (see Appendix B). Similarly to the case of conventional electromagnetic or optical waveguides, the profile along the film normal of a spin-wave mode propagating in a film plane with a wave vector $\boldsymbol{q}_{\|}$can be described as a superposition of two partial plane waves with transverse wave numbers $\kappa_{1}$ and $\kappa_{2}$ at the same frequency $\omega=\omega\left(\boldsymbol{q}_{\|}, \kappa_{1}\right)=\omega\left(\boldsymbol{q}_{\|}, \kappa_{2}\right)$ (see Fig. 1). The in-plane spin-wave dispersion, however, is anisotropic with a minimum frequency corresponding to the wave propagation along the static magnetization (see Appendix C). For the case of an in-plane magnetization, this minimum is achieved at $\kappa=0$, and the dependence of the frequency on the wave number $\kappa$ is symmetric: two partial waves are contrapropagating with equal-by-magnitude but opposite-by-sign wave numbers $\kappa_{1}=-\kappa_{2}$ [see Fig. 1(a)]. Therefore, the corresponding mode profile is represented by a classical standing-wave pattern [see the sketch in Fig. 1(a)]. The situation changes when the sample is magnetized obliquely [see Fig. 1(b)]. In this case, the minimum spin-wave frequency corresponds to a certain $\kappa \neq 0$ value, and the dependence $\omega=\omega\left(\boldsymbol{q}_{\|}, \kappa\right)$ is asymmetric. Thus, at some frequencies the mode profiles are formed by copropagating partial waves. Their superposition results in a traveling-wave pattern, which carries angular momentum and thus can be treated as a transversal spin current $i_{\mathrm{s}}$ directed along the film normal.

It is worth noting that in the framework of the model of a free film (in the case of the absence of spin sinks on the film surfaces), there is no energy flow along the film normal. Moreover, regarding the in-plane wave vector $+\boldsymbol{q}_{\|}$, which is assumed here to be set by the excitation scheme, spin-wave modes with positive and negative wave vectors $\pm \boldsymbol{q}_{\|}$ carry opposite transversal spin currents. Thus, in the case of a

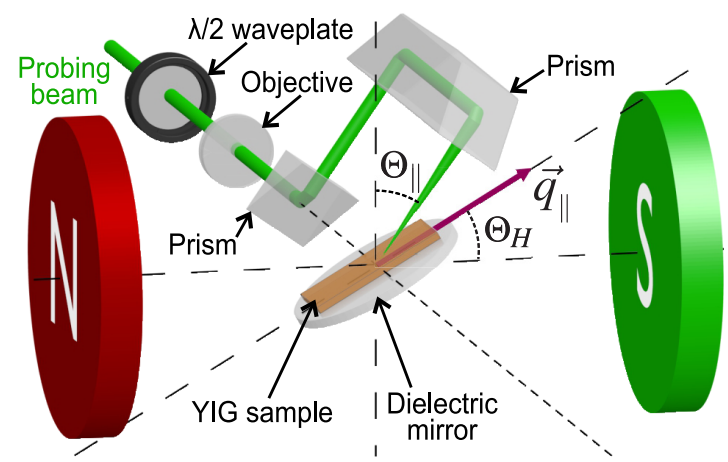

FIG. 2. Experimental BLS setup with wave-vector resolution. A probing laser beam is focused onto the sample, which is placed on a dielectric mirror. A system of prisms allows for variation of the light incidence angle without shift in the focal spot position. The inelastically scattered light is collected in the direction of the incident beam, thus realizing wave-vector resolution (see Appendix B). The dielectric mirror is mounted on a piezodriven rotary stage, which allows for the controlled variation of the angle of magnetization $\Theta_{H}$ in the range from $0^{\circ}$ (in-plane magnetization) to $90^{\circ}$ (out-of-plane magnetization).

symmetric spin-wave excitation or under thermal equilibrium, the net current density $i_{\mathrm{S}}$ is zero. In view of these facts, the following question arises: Would it be possible, in principle, to reveal the existence of such a current in a magnetic film, which is free from any type of a spin sink?

\section{EXPERIMENTAL TECHNIQUE}

Here, we show that the investigation of this fundamental phenomenon is possible in a Brillouin light-scattering (BLS) experiment. The idea behind it is that any modification of the spin-wave profiles along the film normal changes the BLS cross section regardless of the positive or negative orientation of the wave vector $\boldsymbol{q}_{\|}$. In the present work, we use this fact to demonstrate the predicted emergence of the unconventional transversal spin current in an obliquely magnetized thermally excited YIG sample.

The spin-wave spectra are studied at room temperature in a low-damping ferrimagnetic film of yttrium iron garnet (YIG, $\mathrm{Y}_{3} \mathrm{Fe}_{5} \mathrm{O}_{12}$ ) [29-31] by means of BLS spectroscopy with frequency and wave-vector resolution (see Fig. 2). The YIG film sample of a thickness of $L=5.6 \mu \mathrm{m}$ is biased by the magnetic field $H_{0}=2500 \mathrm{Oe}$, which is sufficient to magnetize the sample with a saturation magnetization of $4 \pi M_{\mathrm{s}}=1750 \mathrm{G}$ even when the bias field is perpendicular to the YIG film surface.

The experimental setup, which consists of a YIG film and a wave-vector-resolving BLS system, is schematically shown in Fig. 2 (for a detailed description of the setup and techniques, see Appendixes A and B). The sample is placed on top of a dielectric mirror, which ensures a perfect reflection of the scattered light, and thus significantly enhances the wavenumber selectivity and sensitivity of the experimental setup, allowing for detection of thermal spin waves. In addition, this mirror, being dielectric, does not change the magnetic boundary conditions at the YIG film surface, and thus it does 

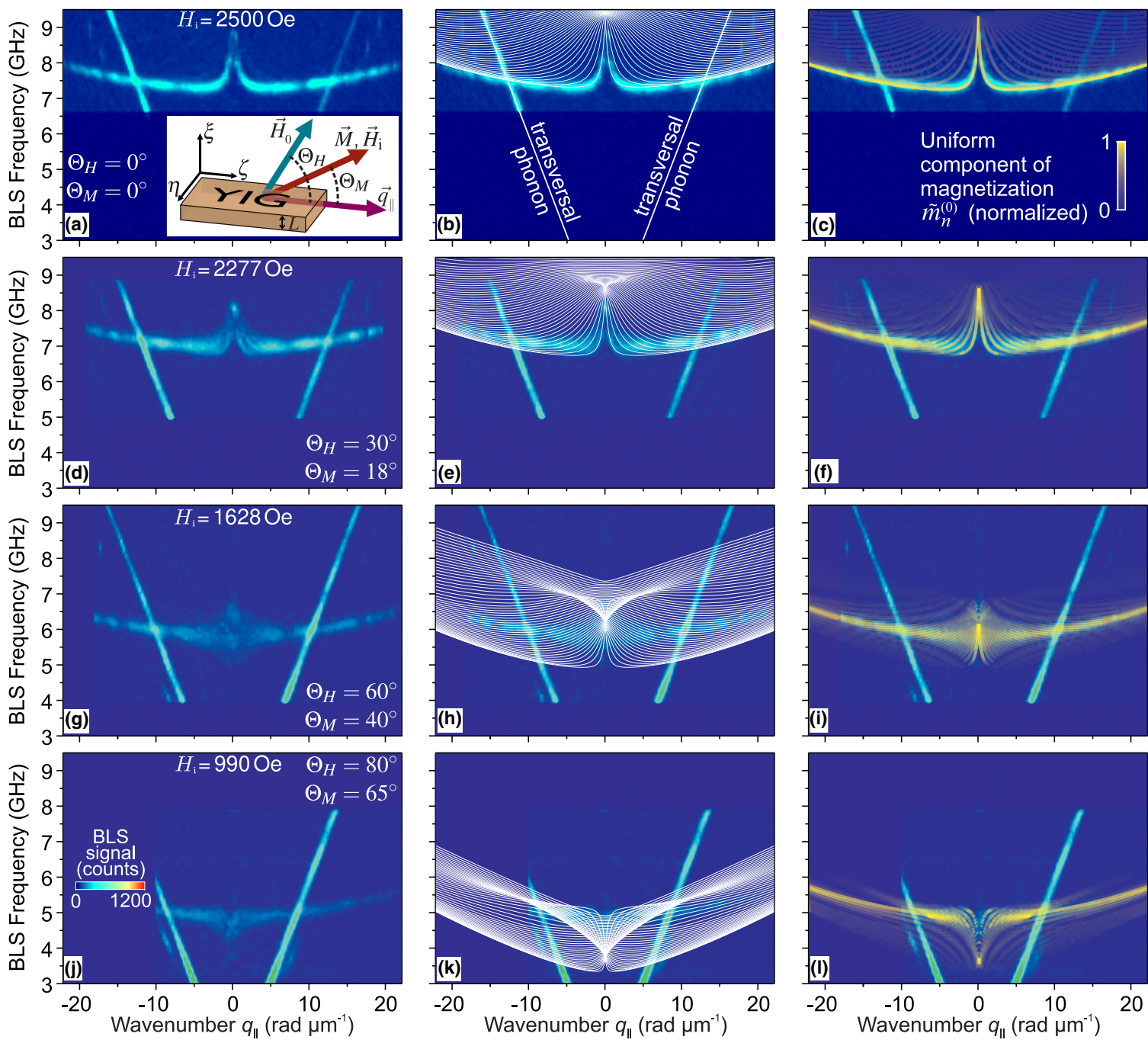

FIG. 3. Spectra. Thermal spin-wave spectra in a 5.6- $\mu$ m-thick YIG film sample measured using a BLS setup with a wave-vector resolution (see Fig. 2) at different magnetization angles $\Theta_{H}$. Shown is the measured BLS intensity (color code) as a function of frequency (vertical scale) and transferred wave vector (horizontal scale). The inset in (a) shows the magnetization geometry. (a) $-(\mathrm{c}) \Theta_{H}=0^{\circ}$; (d) $-(\mathrm{f}) \Theta_{H}=30^{\circ}$; (g) $-(\mathrm{i})$ $\Theta_{H}=60^{\circ}$; (j)-(l) $\Theta_{H}=80^{\circ}$. The panels in the left column demonstrate the raw measured spin-wave spectra. In the panels presented in the middle column, the same data are shown together with the theoretically calculated dispersion curves for the first 50 thickness modes. The images in the right column show the experimentally measured spectra together with the calculated curves of the BLS spectral visibility defined as the normalized spatially uniform component of the profile along the film normal of a particular spin-wave mode.

not modify the spectra of the spin waves, as can occur in the conventional case of a metal mirror [32,33]. The developed setup is placed inside a magnet gap and allows for spin-wave measurements in the full range of the magnetization angle $\Theta_{H}$ from $0^{\circ}$ (in-plane magnetization) to $90^{\circ}$ (out-of-plane magnetization).

\section{SPIN-WAVE SPECTRA AND MODE PROFILES}

Next, we study the thermally excited spin-wave spectrum as a function of $\Theta_{H}$. With increasing $\Theta_{H}$, both the magnitude and the direction of the internal bias magnetic field $\boldsymbol{H}_{i}$ change because of the influence of the demagnetizing field. The angle $\Theta_{M}$ of the internal magnetization $\boldsymbol{M}$ and the magnitude of the internal magnetic field $H_{i}$ can be found from the electrodynamic boundary conditions at the surface of the
YIG film [34]. The values of $H_{i}$ and $\Theta_{M}$ calculated for the particular values of $\Theta_{H}$ used in our experiments are given in Figs. 3(a), 3(d) 3(g), and 3(j).

The observed thermal spectra of dipole-exchange spin waves superimposed by phonon branches are shown in Fig. 3. Panels (a), (d), (g), and (j) in the left column of Fig. 3 show maps of the raw BLS intensity obtained from the roomtemperature populations of magnons and phonons propagating in the YIG film along the projection of the bias magnetic field onto the film plane as functions of the magnon (or phonon) wave vector $\boldsymbol{q}_{\|} \| \boldsymbol{H}_{0}$.

For the in-plane magnetization case $\left[\Theta_{H}=0^{\circ}\right.$, Fig. 3(a)], the experimentally visible spin-wave spectrum consists mainly of the lowest mode of the dipole-exchange backward volume waves [35]. The interplay of dipolar and exchange interactions is clearly visible: the initial dipolar decrease 
of the spin-wave frequency in the long-wavelength section of the spectrum is superseded by the exchange-determined frequency increase at larger wave numbers $\pm q_{\|}$. The steep straight dispersion branches also seen in the data correspond to the transverse acoustic phonons [22,36-39].

The variation of the magnetization angle $\Theta_{H}$ leads to a modification of the observed spectra [see panels (d), (g), and (j) in Fig. 3]. For $\Theta_{H}=30^{\circ}$ shown in Fig. 3(d) one can see that several dispersion branches with negative group velocities $v_{\mathrm{g}}<0$ become visible in the dipolar (long-wavelength) region of the spectrum $\left(\left|q_{\|}\right|<1 \mathrm{rad} \mu \mathrm{m}^{-1}\right)$. With a further increase in $\Theta_{H}$, the contrast of the BLS map in this region decreases, while the exchange magnons and transverse phonons are still clearly visible. The shape of the blurred part of the spin-wave spectrum [see Fig. 3(g) near $q_{\|}=0$ ] provides a hint as to the presence of modes with both positive and negative group velocities $v_{\mathrm{g}}$ in the spin-wave spectrum (both backward and forward types of dipole-exchange magnons [35,40]). At the angle of magnetization of $\Theta_{H}=80^{\circ}$, only magnon modes with $v_{\mathrm{g}} \gtrsim 0$ are seen with rather low contrast in the dipolar area of the spectrum, and all these modes merge into a single exchange-dominated mode at large wave numbers $\left(\left|q_{\|}\right|>\right.$ $\left.1 \mathrm{rad} \mu \mathrm{m}^{-1}\right)$. No spin waves are detected at $\Theta_{H}=90^{\circ}$ : the intensity of the spin-wave maps becomes smaller with an increase of the magnetization angle, and it approaches zero when the magnetization becomes exactly perpendicular to the film plane due to the corresponding decrease in the BLS cross section [41].

Since our BLS setup is sensitive only to the modes with rather uniform profiles along the film normal, the observed blurring of the spin-wave spectrum for $\Theta_{H} \neq 0^{\circ}$ suggests a substantial transformation of the spin-wave mode profiles when compared to the case of in-plane magnetization. To understand this transformation, we further developed the formalism described in Ref. [42] (see Appendix D). Both the dispersion characteristics $\omega_{n}\left(q_{\|}\right)$and the profiles along the film normal $\boldsymbol{m}_{n}(\xi)$ of the spin-wave modes were determined as a function of the in-plane wave number $\pm q_{\|}$and the mode number $n$ [for the used coordinate system, please see the inset in Fig. 3(a)].

The theoretically calculated spin-wave dispersion characteristics plotted by white lines are presented together with the experimental data in the central column of Fig. 3 [panels (b), (e), (h), and (k)]. It can be clearly seen that the calculation describes the fundamental spin-wave mode in the case of in-plane magnetization very well [Fig. 3(b)], but the higherorder modes present in the theory are not visible in the experiment. At magnetization angles $\Theta_{H}>0^{\circ}$, the situation becomes worse since many of the theoretically predicted spinwave modes are not observed in our BLS experiment [see panels (e), (h), and (k) in Fig. 3]. This apparent discrepancy is related to the aforementioned fact that the BLS cross section is sensitive only to the modes having a uniform component in their profiles along the film normal. Thus, the spin-wave modes with profiles oscillating about zero along the film normal do not contribute to the BLS signal.

The calculated profiles of two spin-wave modes are exemplarily shown in Fig. 4 for a few selected wave numbers $q_{\|}$. The spatially uniform zero Fourier component $\left(\tilde{\boldsymbol{m}}_{n}^{(0)} ;\right.$ see

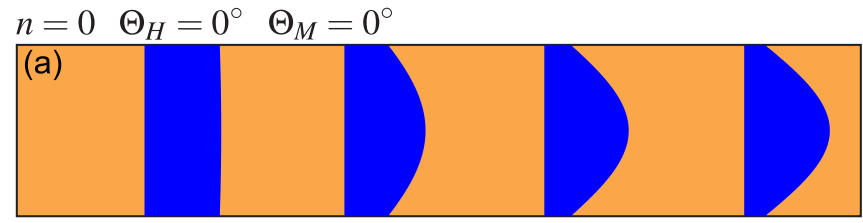

$n=21 \Theta_{H}=0^{\circ} \quad \Theta_{M}=0^{\circ}$

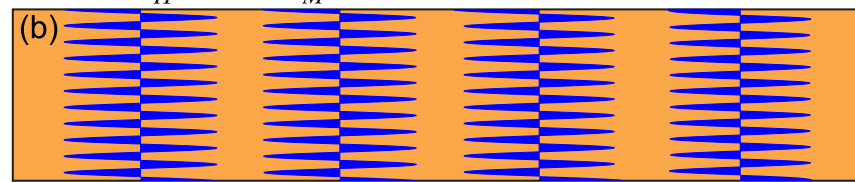

$n=0 \quad \Theta_{H}=60^{\circ} \Theta_{M}=40^{\circ}$

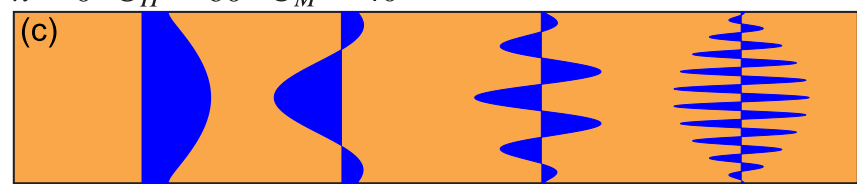

$n=21 \Theta_{H}=60^{\circ} \Theta_{M}=40^{\circ}$

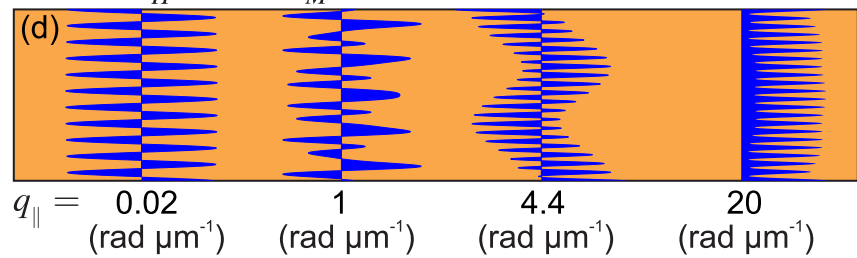

FIG. 4. Mode profiles. (a)-(d) Numerically calculated profiles along the film normal of the spin-wave modes in a 5.6- $\mu$ m-thick YIG film sample.

Appendix D for details) of each such profile is directly proportional to the sensitivity of the BLS setup to the corresponding spin-wave mode. The magnitudes of this component are presented in the right column of Fig. 3 [panels (c), (f), (i), and (l)] by yellow lines jointly with the BLS intensity maps. As can be seen, these yellow lines agree very well with the experimental data [compare panels (c), (f), (i), and (1) in Fig. 3 to panels (a), $(\mathrm{d}),(\mathrm{g})$, and $(\mathrm{j})$ in the same figure]. The fundamental $(n=0)$ mode has a perfectly uniform profile along the film normal at small $\left(q_{\|} L<1\right)$ in-plane wave numbers $q_{\|}=0.02 \mathrm{rad} \mu \mathrm{m}^{-1}$, and it is therefore very visible in the BLS experiment. With the increase in $q_{\|}$, the effective pinning in the profile along the film normal of this mode gradually increases. This pinning is not at all related to any surface anisotropy at the film boundaries, but it has a purely dipolar character [43,44]. Note also that the higher-order (e.g., $n=21$ ) modes have oscillating profiles along the film normal, and therefore they cannot be detected in the BLS experiment.

The mode profiles in an obliquely magnetized magnetic film (for illustration, we consider here the case of $\Theta_{H}=60^{\circ}$ ) have rather unusual shapes [see Figs. 4(c) and 4(d)]. The profile along the film normal of the $n=0$ mode at relatively small wave-number values $q_{\|}=0.02 \mathrm{rad} \mu \mathrm{m}^{-1}$ is rather uniform in comparison to the profiles shown in Fig. 4(b), and this mode is therefore clearly visible in the BLS experiment [similar to the situation in Fig. 4(a)]. However, with an increase in $q_{\|}$, the mode profile acquires more and more oscillations along the film normal, leading to a lower visibility of these modes in the experiment [see Fig. 3(g)]. At $q_{\|}>1 \mathrm{rad} \mu \mathrm{m}^{-1}$, the visibility of these modes vanishes completely. The 
opposite situation occurs for the higher-order mode with $n=$ 21 [see Fig. 4(d)]. The profile along the film normal of such a mode, which is purely oscillating at $q_{\|}=0.02 \mathrm{rad}_{\mu \mathrm{m}}^{-1}$, continues to oscillate at higher values of the in-plane wave number but, surprisingly enough, also possesses a nonzero uniform component [see Fig. 4(d) for $q_{\|}=20 \mathrm{rad}_{\mu \mathrm{m}}^{-1}$ ]. It is worth noting that the above-described transformation of the mode profiles along the film normal is not exclusively related to the case of a plain YIG film, but it can also explain the effects observed in permalloy nanodots [45-47] and magnetic multilayers [48].

\section{TRANSVERSAL SPIN CURRENTS}

The unusual profiles of the spin-wave modes presented in Figs. 4(c) and 4(d) find a natural qualitative explanation in the framework of the proposed model of transversal spin currents.

In the case of an in-plane magnetized film [Fig. 1(a)], which is characterized by the symmetric dependence $\omega(\kappa)$ for positive and negative $\kappa$ directions, the mode profiles demonstrate perfect standing-wave patterns along the normal of the film [see Figs. 4(a) and 4(b)]. The fundamental mode with the lowest possible frequency has $\left|\kappa_{1,2}\right| \approx 0$, i.e., its profile is quasiuniform [see Fig. 4(a)]. With increasing frequency, the transversal wave numbers $\left|\kappa_{1,2}\right|$ also increase, and therefore the number of nodes in the mode's profile along the film normal (the mode's index $n$ ) also increases, as is visible in Fig. 4(b).

The situation is different for an obliquely magnetized film. Due to the anisotropic character of the dipolar magnetic interaction $[32,42]$, the dependence $\omega(\kappa)$ is no longer symmetric [see Fig. 1(b)]. Now, for spin waves with relatively long wavelength, the minimum frequency is achieved when their total wave vector $\boldsymbol{q}$ is parallel to the internal magnetic field, i.e., for $\kappa=\kappa_{\min } \approx q_{\|} \tan \Theta_{M}$. Therefore, in this case, the fundamental mode will be formed by two partial waves $\kappa_{1} \approx \kappa_{2} \approx$ $\kappa_{\min }$ and possesses approximately $\kappa_{\min } L / \pi \approx\left(q_{\|} L / \pi\right) \tan \Theta_{M}$ nodes along the film normal. We would like to underline the fact that in the shown case, both partial waves have transverse wave vectors of the same direction, so the resultant mode has a quasitraveling character, but the group velocities of the partial waves have opposite signs, which means that the mode does not transfer energy along the film normal. The increase of the number of nodes of the fundamental mode with an increase in $q_{\|}$is clearly seen in Fig. 4(c). With the increase in the mode number $n$, the transversal wave number $\kappa_{1}$ decreases while $\kappa_{2}$ increases [see Fig. 1(b)], producing the nontrivial patterns seen in Fig. 4(d). In particular, for a certain higher-order mode (or, in the case of a fixed mode number $n$, for a particular in-plane wave vector $\left.q_{\|}\right), \kappa_{1} \approx 0$, and the mode attains a comblike profile seen in Fig. 4(d) for $q_{\|}=20 \mathrm{rad} \mu \mathrm{m}^{-1}$.

The above considerations lead to the essential conclusion, namely that the profiles along the film normal of the magnon modes propagating in an obliquely magnetized magnetic film do not fully form standing-wave patterns, but they have a traveling character. This conclusion is further confirmed by a direct visualization of the time evolution of the mode profiles shown in the supplemental movie [49].

This unconventional traveling nature of the magnon mode's profiles has important implications in the field of modern spintronics. In particular, by direct evaluation, one can show that these modes carry a nonzero intrinsic exchange spin current with the density

$$
i_{\mathrm{s}} \propto\left|\boldsymbol{m}_{n} \times \partial \boldsymbol{m}_{n}^{*} / \partial \xi\right|
$$

in the direction perpendicular to the film plane. From the point of view of the symmetry considerations, the appearance of the perpendicular spin current is associated with the symmetry breaking induced by the oblique magnetization and the direction of the in-plane wave vector of the spin waves, $\boldsymbol{q}_{\text {. }}$. Reversing either the direction of the bias magnetic field or the direction of $\boldsymbol{q}_{\|}$will reverse the sign of the transverse spin current. In particular, the spin waves with $q_{\|} \simeq 0$ always carry zero perpendicular current, so the effects related to this current require traveling spin waves, and they cannot be seen in a ferromagnetic resonance kind of experiment.

It should be remarked that in the state of thermal equilibrium, the magnon modes with wave vectors $+q_{\|}$and $-q_{\|}$ are equally populated, and the transverse spin currents they carry mutually compensate each other. Therefore, the intrinsic transverse spin current does not have any direct influence on the thermal equilibrium properties of a magnetic film.

If a spin wave is excited in a certain direction, let us assume in the $+\zeta$ direction, i.e., if we only have $+q_{\|}$, the effect of a transverse spin current will manifest itself explicitly. In this scenario, we may find different spin pumping efficiencies at the opposite surfaces of the film. This effect, in particular, can be used for the development of nonreciprocal spin-current transmission lines based on propagating spin waves. The contribution of the transversal spin current for the modes with a quasiuniform profile along the film normal is of particular interest [e.g., all profiles in Fig. 4(a) and the profile at $q_{\|}=$ $20 \mathrm{rad} \mu \mathrm{m}^{-1}$ in Fig. 4(d)]. These modes can be excited, for example by microstructured microwave antennas. We have examined the $\Theta_{H}$ versus $q_{\|}$parameter space regarding the transversal spin-current efficiency. The results of numerical calculations of the spin-current magnitude using Eq. (1) are shown in Fig. 5. As can be seen, the maximal transversal spin-current density is achieved at approximately $\Theta_{M} \simeq 55^{\circ}$ and $q_{\|} \simeq 5 \operatorname{rad} \mu \mathrm{m}^{-1}$, which corresponds to the wavelength of $1.2 \mu \mathrm{m}$ and fits well to the requirements of modern nanoscale magnonic devices. As the described spin currents along the film normal originate from the interplay between dipolar and exchange interactions, they vanish in the case of pure exchange waves as well as for pure dipolar waves. As expected, $i_{\mathrm{s}}$ weakens with an increase in the in-plane wave number $q_{\|}$and becomes zero at the limiting values $\left(0^{\circ}\right.$ and $90^{\circ}$ ) of the magnetization angle $\Theta_{M}$ (see Fig. 5).

\section{DISCUSSION}

One important issue that should be discussed here is the question about the source of the transversal spin current. It may sound paradoxical, but the source of this exchange spin current is the magnetic dipolar interaction, which couples the spin and lattice degrees of freedom of the studied magnetic sample. In a magnetic system with dipole interaction, only the total angular momentum, which consists of the spin and mechanical parts, is conserved [50,51]. This fact has a number of experimentally observable and well-understood 


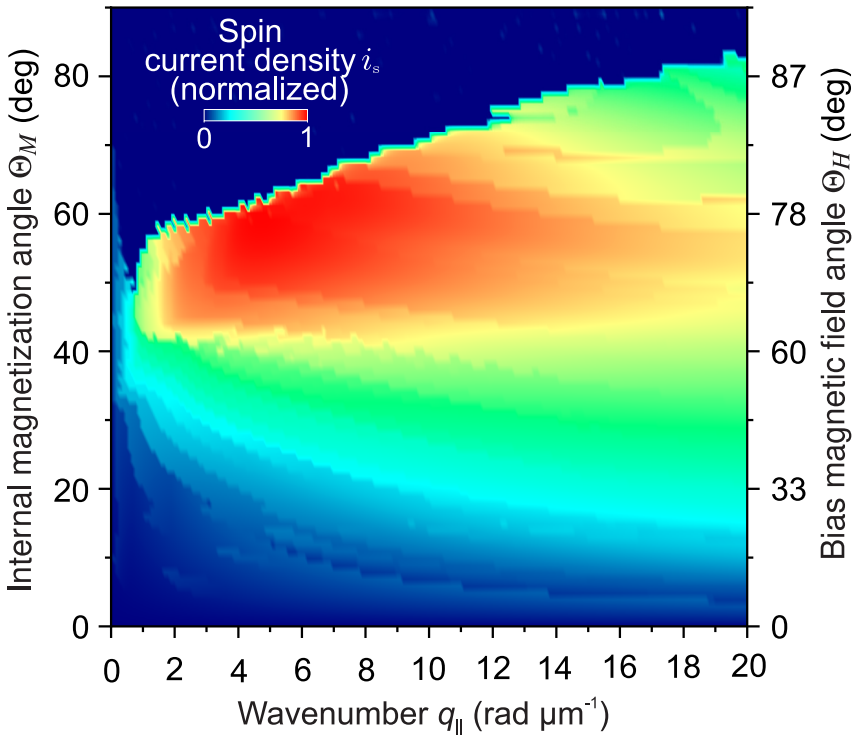

FIG. 5. Spin current. Color-coded density of the spin current $i_{\mathrm{s}}$ calculated using Eq. (1) as a function of the in-plane wave number $q_{\|}$and magnetization angle $\Theta_{M}$ for the modes with quasiuniform distribution along the film normal.

manifestations (see, for example, Refs. [52,53]), such as, e.g., the parallel pumping process [54], when one photon carrying zero angular momentum splits into two magnons with total spin quantum number 2; the first-order Suhl instability [51,55], when one magnon splits in two; or the Einstein-de Haas effect [56], when a magnetization change of a free ferromagnetic body causes this body to rotate.

From a rigorous mathematical point of view, the effect of the long-range dipolar interaction cannot be described as a certain current term, which would be able to compensate for the exchange spin-current density $i_{\mathrm{s}}$, calculated with Eq. (1), at every point in space. It should rather be represented in the form of an additional spin sink or spin source (see Appendix E for details). Such a rigorous mathematical treatment is necessary for the proper description of spin dynamics in all the above-mentioned examples, as well as in our case, when the transversal spin-current density $i_{\mathrm{s}}$ in Fig. 5 is calculated for the spin-wave modes with quasiuniform distribution along the film normal. Furthermore, the lack of the pointwise balance between the angular momenta of a different nature should allow for a separate detection of the mechanical and spinrelated phenomena.

In the case of an external excitation of the spin-wave modes carrying the transversal spin current, the angular momentum conservation law would lead to mechanical deformations and/or to the rotation of the magnetic film as a whole, which could be potentially detected using the existing methods of spin mechatronics [57]. Simultaneously, the presence of the unconventional spin current may be directly electrically detected in the following proposed experiment. In the case of oblique magnetization, spin waves propagating in opposite in-plane directions should have different spin-pumping efficiencies at the surfaces of the magnetic film covered with a few-nanometer-thick nonmagnetic heavy metal. The difference in the values of spin currents injected by the con- trapropagating waves to such a spin sink can be measured as a corresponding difference in the inverse spin Hall voltages generated across the metal layer [59]. However, the excitation of short-wavelength spin waves $\left(\left|q_{\|}\right|>4 \mathrm{rad} \mu \mathrm{m}^{-1}\right)$, for which this effect is expected to be sufficiently high (see Fig. 5), is a rather difficult task due to the absence of reliable unidirectional spin-wave emitters. Development of such emitters is a work in progress $[58,60,61]$.

We believe that these interesting and nontrivial properties of the spin-wave modes in obliquely magnetized magnetic films will be used in the future to control the effects of spin pumping and to generate spin currents in nanoscale spintronic signal processing and signal-generating devices. Moreover, similar effects should also be observed in photonic and acoustic waveguide structures made of anisotropic materials, in the case when the direction of the main crystallographic axis does not coincide with the direction of wave propagation.

\section{ACKNOWLEDGMENTS}

We are indebted to R. V. Verba and P. Pirro for discussions. Funding by the Deutsche Forschungsgemeinschaft (DFG, German Research Foundation) within the Research Units TRR 49 (Project A07) and TRR 173-268565370 "Spin+X" (Projects B01 and B04) as well as financial support by the European Research Council within the Advanced Grant 694709 SuperMagnonics-“Supercurrents of Magnon Condensates for Advanced Magnonics" is gratefully acknowledged. This work was also supported in part by the US National Science Foundation (Grants No. EFMA-1641989 and No. ECCS-1708982), by the U.S. Air Force Office of Scientific Research under the MURI Grant No. FA9550-19-1-0307, and by the Oakland University Foundation.

\section{APPENDIX A: SAMPLE}

The yttrium iron garnet ( $\mathrm{YIG}, \mathrm{Y}_{3} \mathrm{Fe}_{5} \mathrm{O}_{12}$ ) sample is $5 \mathrm{~mm}$ long and $1 \mathrm{~mm}$ wide. The single-crystal YIG film of $5.6 \mu \mathrm{m}$ thickness has been grown in the (111) crystallographic plane on a gadolinium gallium garnet $\left(\mathrm{GGG}, \mathrm{Gd}_{3} \mathrm{Ga}_{5} \mathrm{O}_{12}\right)$ substrate by liquid-phase epitaxy.

\section{APPENDIX B: BRILLOUIN LIGHT-SCATTERING SPECTROSCOPY}

The spin-wave spectrum was visualized by means of wavevector-resolved BLS spectroscopy [62] in backscattering geometry. Brillouin light-scattering spectroscopy can be understood as the diffraction of the probing light from a moving Bragg grating produced by a spin-wave mode [63]. As a result, some portion of the scattered light is shifted in frequency by the frequency of this mode. In addition, the diffraction from the grating leads to a transfer of momentum during this process. For example, the in-plane component of the wave vector $\boldsymbol{q}_{\mathrm{L}}$ of the incident light is inverted by a spin-wave mode propagating along the projection of a probing beam on a thin magnetic film if the spin-wave wave number $q$ satisfies the Bragg condition $q=-2 q_{\mathrm{L}} \sin \left(\Theta_{\|}\right)$, where $\Theta_{\|}$is the angle of laser light incidence. By changing the angle $\Theta_{\|}$, wave-vector 
selection of in-plane spin waves with wave vector $\boldsymbol{q}$ can be implemented.

In our experiment, the probing laser beam of $532 \mathrm{~nm}$ wavelength generated by a single-mode solid-state laser is focused by a precise objective onto the YIG film sample. The probing beam is steered by a system of prisms, enabling rotation of the probing laser beam around the sample. The optomechanical system is precisely aligned to keep the position of the probing spot at the same place at the sample surface while changing the angle of incidence $\Theta_{\|}$. In such a setup, the rotation angle $\Theta_{\|}$(see Fig. 2) and, consequently, the magnitude of the probed magnon wave vector $\boldsymbol{q}$ are limited only by the wavelength $\lambda$ of the probing laser light (for $\lambda=532 \mathrm{~nm}, q_{\|}^{\max }=2 q_{\mathrm{L}}=$ $23.6 \mathrm{rad} \mu \mathrm{m}^{-1}$ ), and are not constrained by geometry of the magnet, as was the case in Ref. [62]. The polarization of the incident light was held parallel to the surface of the sample by utilizing a $\lambda / 2$ wave plate, which is rotated simultaneously with the rotation of the prisms. The whole beam steering system is placed directly between the poles of the electromagnet, ensuring high-field uniformity and stability. The sample is placed with the YIG side on top of a broadband dielectric mirror (commercially available "Thorlabs" E02 multilayer mirror for the 400-700 nm light wavelength). As it was checked in test experiments, this mirror does not affect the phonon and spin-wave dispersions. Its role is to reflect the inelastically scattered light, whose in-plane wave-vector component is inverted by a spin-wave mode, back through the objective for further frequency analysis. The dielectric mirror is fixed on a piezodriven nonmagnetic rotary stage, which was used to change the angle $\Theta_{H}$ between the bias magnetic field and the film surface.

The light collected by the objective is directed to a multipass tandem Fabry-Pérot interferometer [64-66] for frequency selection. At the output of the interferometer, a single photon counting avalanche diode detector is placed. The output of the detector is connected to a counter module. Every time the detector registers a photon, this event is recorded to a database that collects the number of arrived photons. The frequency of the interferometer's transmission is also recorded, thus providing frequency information for each detected photon.

\section{APPENDIX C: SIMPLIFIED MODEL OF THE DIPOLE-EXCHANGE SPIN-WAVE SPECTRUM}

Considering the Landau-Lifshitz and magnetostatic Maxwell equations inside a magnetic film, one can show that a propagating magnon mode can be represented by the sum of several partial plane waves with a total wave vector $\boldsymbol{q}=q_{\Downarrow} \boldsymbol{e}_{\zeta}+\kappa \boldsymbol{e}_{\xi}$, where $\boldsymbol{e}_{\zeta}$ and $\boldsymbol{e}_{\xi}$ are unit vectors along the in-plane propagation direction and perpendicular to the film plane, respectively [see Fig. 1 and the inset in Fig. 3(a)]. The relation between the total wave vector $\boldsymbol{q}$ and the mode frequency $\omega$ is the same as in the case of an infinite magnetic medium:

$$
\omega_{q}^{2}=\left(\omega_{H}+\omega_{M} \lambda_{\text {ex }}^{2} q^{2}\right)\left(\omega_{H}+\omega_{M} \lambda_{\text {ex }}^{2} q^{2}+\omega_{M} \sin ^{2} \theta_{q}\right) .
$$

Here $\omega_{H}=\gamma H_{i}, \omega_{M}=\gamma 4 \pi M_{\mathrm{s}}, \gamma$ is the gyromagnetic ratio, $\lambda_{\text {ex }}$ is the exchange length, and $\sin ^{2} \theta_{q}=1-\left(q_{\|} \cos \Theta_{M}+\right.$ $\left.\kappa \sin \Theta_{M}\right)^{2} / q^{2}$, where $\theta_{\boldsymbol{q}}$ is the angle between $\boldsymbol{q}$ and $\boldsymbol{M}$. Equation $(\mathrm{C} 1)$ determines the allowed values of the perpendicular wave-vector component $\kappa$, and is, effectively, a sixth-order equation in $\kappa$. In the frequency range of propagating magnon modes, two solutions of Eq. (C1), $\kappa_{1}$ and $\kappa_{2}$, are real, while the other four solutions have nonzero imaginary parts. The complex solutions of Eq. (C1) have the absolute values $|\kappa| \sim$ $1 / \lambda_{\text {ex }}$, and they describe surface corrections to the profile along the film normal of the considered spin-wave mode. An account of these evanescent partial waves is necessary for the analysis of boundary conditions at the film surfaces. However, their influence is limited only to a rather thin boundary layer of the width $\Delta \xi \approx 1 /|\kappa| \sim \lambda_{\text {ex }}$. In a magnetic film of a finite thickness $L$, the profile of a spin-wave mode is accurately described outside from a thin boundary layer of the width $\Delta \xi \approx 1 /|\kappa| \sim \lambda_{\text {ex }}$ by the sum of two frequency-degenerate partial waves with the real-valued perpendicular wave-vector components $\kappa_{1}$ and $\kappa_{2}$.

\section{APPENDIX D: SPIN-WAVE SPECTRA AND MODE PROFILES CALCULATION}

We consider a ferromagnetic film of finite thickness $L$ in the $\xi$ direction [see the inset in Fig. 3(a)] that is infinite in the two in-plane directions. We assume that a nonuniform spinwave mode of vector amplitude $\boldsymbol{m}_{n}(\xi)$ is propagating along the $\zeta$ axis lying in the film plane, and that this axis is parallel to the projection of the obliquely applied bias magnetic field $\boldsymbol{H}_{0}$ onto the film plane [see inset in Fig. 3(a)]:

$$
\boldsymbol{m}(\xi, \zeta, t)=\boldsymbol{m}_{n}(\xi) \exp \left[i\left(\omega_{n} t-q_{\|} \zeta\right)\right],
$$

where $\omega_{n}=\omega_{n}\left(q_{\|}\right)$is the frequency of the $n$th spin-wave mode.

Following the method used in Ref. [42], we expand the spin-wave mode profile along the film normal $\boldsymbol{m}_{n}(\xi)$ into an infinite series of complete orthogonal functions, which are the eigenfunctions of the second-order exchange differential operator and the exchange boundary conditions corresponding to zero surface anisotropy (unpinned surface spins):

$$
\boldsymbol{m}_{n}(\xi)=\sum_{j} \tilde{\boldsymbol{m}}_{n}^{(j)} \cos \left[\frac{\pi j}{L}\left(\xi+\frac{L}{2}\right)\right] .
$$

Using Eq. (D2) in the framework of the formalism of Ref. [42], it is possible to reduce the Landau-Lifshitz equation describing the spin-wave dynamics in a magnetic film to the following infinite system of algebraic equations for the vector coefficients $\tilde{\boldsymbol{m}}_{n}^{(j)}$ :

$$
i \frac{\omega_{n}}{\omega_{\mathrm{M}}} \tilde{\boldsymbol{m}}_{n}^{(j)}=\sum_{j^{\prime}} \hat{W}_{j^{\prime}}^{j} \tilde{\boldsymbol{m}}_{n}^{\left(j^{\prime}\right)},
$$

where the square matrix $\hat{W}$ can be found by simple algebraic transformation of Eq. (22) from Ref. [42].

Next, the dispersion characteristics $\omega_{n}\left(q_{\|}\right)$and the profiles along the film normal $\boldsymbol{m}_{n}(\xi)$ of the spin-wave modes can be calculated as solutions of the eigenvalue problem of Eq. (D3). 


\section{APPENDIX E: EFFECTS OF THE EXCHANGE AND DIPOLAR INTERACTIONS ON SPIN ANGULAR MOMENTUM}

The density of the spin angular momentum carried by a small-amplitude spin wave can be written as

$$
\mathcal{S}=\frac{\hbar \Delta M_{\mathrm{s}}}{g_{\mathrm{e}} \mu_{\mathrm{B}}} \approx \frac{M_{\mathrm{s}}}{2 \gamma}|\boldsymbol{m}|^{2},
$$

where $\hbar$ is the reduced Planck constant, $g_{\mathrm{e}}$ is the spectroscopic Landé factor, $\mu_{\mathrm{B}}$ is the Bohr magneton, $\Delta M_{\mathrm{s}}$ is the reduction of the static magnetization $M_{\mathrm{S}}$ caused by the precession of the magnetic moment $\boldsymbol{M}(t, \boldsymbol{r})$, and $\boldsymbol{r}$ is the position vector. $\boldsymbol{m}(t, \boldsymbol{r}) \approx \boldsymbol{M}(t, \boldsymbol{r}) / M_{\mathrm{s}}-\boldsymbol{m}_{0}$ is the dimensionless vector of magnetization deviations from its equilibrium direction $\boldsymbol{m}_{0}$. Using the Landau-Lifshitz equation of magnetization dynamics, it is possible to obtain the exact equation describing the dynamics of the spin density:

$$
\frac{\partial \mathcal{S}}{\partial t}+\nabla \cdot \boldsymbol{i}_{\mathrm{s}}=\mathcal{Q}
$$

Here $\boldsymbol{i}_{\mathrm{s}}$ is the vector with the components

$$
\boldsymbol{i}_{\mathrm{s}}^{(j)}=A_{\mathrm{ex}} \boldsymbol{m}_{0} \cdot\left(\frac{\partial \boldsymbol{m}}{\partial r_{j}} \times \boldsymbol{m}\right),
$$

$A_{\text {ex }}$ is the exchange constant, and $\mathcal{Q}$ is given by

$$
\mathcal{Q}=M_{\mathrm{s}} \boldsymbol{m}_{0} \cdot\left(\boldsymbol{m} \times \boldsymbol{h}_{\mathrm{dip}}\right),
$$

where $\boldsymbol{h}_{\text {dip }}$ is the dynamic dipolar field created by the spin wave.

In Eq. (E2), the term $\nabla \cdot \boldsymbol{i}_{\mathrm{s}}$ originates from the exchange interaction, while the term $\mathcal{Q}$ has a solely dipolar origin.
In the absence of the dipolar interaction $(\mathcal{Q}=0)$, Eq. (E2) has the standard form of a continuity equation and describes conservation of the spin angular momentum of the magnetic subsystem, with $\boldsymbol{i}_{\mathrm{s}}$ being the spin current density.

In a general case, the dipolar term $\mathcal{Q}$ cannot be written as a divergence of a certain vector function, i.e., the effect of the dipolar interaction cannot be described as a "dipolar spin current." Physically, the dipolar interaction couples the spin and spatial degrees of freedom of a magnetic body, and the term $\mathcal{Q}$ describes transfer of the angular momentum between these subsystems. When one is interested only in the magnetic dynamics, the dipolar interaction should be described as a spin source/sink term $\mathcal{Q}$. Since the total angular momentum, which consists of the spin and mechanical parts, is conserved, a similar source/sink term appears in the equation for the $\mathbf{m}_{0}$ component of the mechanical angular momentum, which is equal to $\mathcal{Q}_{\text {mech }}=-\mathcal{Q}$. Note that $\mathcal{Q}_{\text {mech }}$ is the density of the mechanical torque that acts on a magnetic body and can be, in principle, measured experimentally.

In any stationary situation (for example, in the case of propagation of a plane wave), the spin density is constant, $\partial \mathcal{S} / \partial t=0$. Then, Eq. (E2) implies that the exchange and dipolar contributions compensate each other in the sense $\nabla$. $\boldsymbol{i}_{\mathrm{s}}=\mathcal{Q}$. This compensation, however, is, in general, nonlocal and does not exclude experimentally observable spin-current effects. For example, as considered in this work, propagation of a spin wave carrying a transversal spin current would create mechanical torques $\mathcal{Q}_{\text {mech }}$ with opposite signs at the two surfaces of the magnetic film, which would lead to a mechanical deformation of the film. This effect will be present and can be experimentally detected even if the magnetic film is spin-sink-free and the total spin angular momentum of the system remains constant.
[1] A. V. Chumak, V. I. Vasyuchka, A. A. Serga, and B. Hillebrands, Magnon spintronics, Nat. Phys. 11, 453 (2015).

[2] H. Yu, J. Xiao, and P. Pirro, Magnon spintronics, J. Magn. Magn. Mater. 450, 1 (2018).

[3] A. A. Serga, A. V. Chumak, and B. Hillebrands, YIG magnonics, J. Phys. D 43, 264002 (2010).

[4] V. V. Kruglyak, S. O. Demokritov, and D. Grundler, Magnonics, J. Phys. D 43, 264001 (2010).

[5] A. Khitun, M. Bao, and K. L. Wang, Magnonic logic circuits, J. Phys. D 43, 264005 (2010).

[6] C. Liu, J. Chen, T. Liu, F. Heimbach, H. Yu, Y. Xiao, J. Hu, M. Liu, H. Chang, T. Stueckler, S. Tu, Y. Zhang, Y. Zhang, P. Gao, Z. Liao, D. Yu, K. Xia, N. Lei, W. Zhao, and M. Wu, Long-distance propagation of short-wavelength spin waves, Nat. Commun. 9, 738 (2018).

[7] J. Chen, F. Heimbach, T. Liu, H. Yu, C. Liu, H. Chang, T. Stückler, J. Hu, L. Zeng, Y. Zhang, Z. Liao, D. Yu, W. Zhao, and $\mathrm{M}$. Wu, Spin wave propagation in perpendicularly magnetized $\mathrm{nm}$-thick yttrium iron garnet films, J. Magn. Magn. Mater. 450, 3 (2017)

[8] Y. Kajiwara, K. Harii, S. Takahashi, J. Ohe, K. Uchida, M. Mizuguchi, H. Umezawa, H. Kawai, K. Ando, K. Takanashi, S. Maekawa, and E. Saitoh, Transmission of electrical signals by spin-wave interconversion in a magnetic insulator, Nature (London) 464, 262 (2010).

[9] K. Wagner, A. Kákay, K. Schultheiss, A. Henschke, T. Sebastian, and H. Schultheiss, Magnetic domain walls as reconfigurable spin-wave nanochannels, Nat. Nanotechnol. 11, 432 (2016).

[10] Q. Wang, P. Pirro, R. Verba, A. Slavin, B. Hillebrands, and A. V. Chumak, Reconfigurable nanoscale spin-wave directional coupler, Sci. Adv. 4, e1701517 (2018).

[11] A. V. Chumak, A. A. Serga, and B. Hillebrands, Magnonic crystals for data processing, J. Phys. D 50, 244001 (2017).

[12] I. Lisenkov, D. Kalyabin, S. Osokin, J. W. Klos, M. Krawczyk, and S. Nikitov, Nonreciprocity of edge modes in 1D magnonic crystal, J. Magn. Magn. Mater. 378, 313 (2015).

[13] T. Fischer, M. Kewenig, D. A. Bozhko, A. A. Serga, I. I. Syvorotka, F. Ciubotaru, C. Adelmann, B. Hillebrands, and A. V. Chumak, Experimental prototype of a spin-wave majority gate, Appl. Phys. Lett. 110, 152401 (2017).

[14] A. V. Chumak, A. A. Serga, and B. Hillebrands, Magnon transistor for all-magnon data processing, Nat. Commun. 5, 4700 (2014).

[15] T. Schneider, A. A. Serga, A. V. Chumak, C. W. Sandweg, S. Trudel, S. Wolff, M. P. Kostylev, V. S. Tiberkevich, 
A. N. Slavin, and B. Hillebrands, Nondiffractive Subwavelength Wave Beams in a Medium with Externally Controlled Anisotropy, Phys. Rev. Lett. 104, 197203 (2010).

[16] S. O. Demokritov, V. E. Demidov, O. Dzyapko, G. A. Melkov, A. A. Serga, B. Hillebrands, and A. N. Slavin, Bose-Einstein condensation of quasi-equilibrium magnons at room temperature under pumping, Nature (London) 443, 430 (2006).

[17] A. A. Serga, V. S. Tiberkevich, C. W. Sandweg, V. I. Vasyuchka, D. A. Bozhko, A. V. Chumak, T. Neumann, B. Obry, G. A. Melkov, A. N. Slavin, and B. Hillebrands, Bose-Einstein condensation in an ultra-hot gas of pumped magnons, Nat. Commun. 5, 3452 (2014).

[18] M. Schneider, T. Brächer, D. Breitbach, V. Lauer, P. Pirro, D. A. Bozhko, H. Yu. Musiienko-Shmarova, B. Heinz, Q. Wang, T. Meyer, F. Heussner, S. Keller, E. Th. Papaioannou, B. Lägel, T. Löber, C. Dubs, A. N. Slavin, V. S. Tiberkevich, A. A. Serga, B. Hillebrands, and A. V. Chumak, Bose-Einstein condensation of quasiparticles by rapid cooling, Nat. Nanotechnol. (2020).

[19] D. A. Bozhko, A. A. Serga, P. Clausen, V. I. Vasyuchka, F. Heussner, G. A. Melkov, A. Pomyalov, V. S. L'vov, and B. Hillebrands, Supercurrent in a room-temperature Bose-Einstein magnon condensate, Nat. Phys. 12, 1057 (2016).

[20] K. Nakata, K. A. van Hoogdalem, P. Simon, and D. Loss, Josephson and persistent spin currents in Bose-Einstein condensates of magnons, Phys. Rev. B 90, 144419 (2014).

[21] K. Nakata, P. Simon, and D. Loss, Magnon transport through microwave pumping, Phys. Rev. B 92, 014422 (2015).

[22] D. A. Bozhko, P. Clausen, G. A. Melkov, V. S. L'vov, A. Pomyalov, V. I. Vasyuchka, A. V. Chumak, B. Hillebrands, and A. A. Serga, Bottleneck Accumulation of Hybrid MagnetoElastic Bosons, Phys. Rev. Lett. 118, 237201 (2017).

[23] S. Takei and Y. Tserkovnyak, Superfluid Spin Transport Through Easy-Plane Ferromagnetic Insulators, Phys. Rev. Lett. 112, 227201 (2014).

[24] C. Sun, T. Nattermann, and V. L. Pokrovsky, Unconventional Superfluidity in Yttrium Iron Garnet Films, Phys. Rev. Lett. 116, 257205 (2016).

[25] H. Skarsvåg, C. Holmqvist, and A. Brataas, Spin Superfluidity and Long-Range Transport in Thin-Film Ferromagnets, Phys. Rev. Lett. 115, 237201 (2015).

[26] Y. Kajiwara, S. Takahashi, S. Maekawa, and E. Saitoh, Detection of spin-wave spin current in a magnetic insulator, IEEE Trans. Magn. 47, 1591 (2011).

[27] A. V. Chumak, A. A. Serga, M. B. Jungfleisch, R. Neb, D. A. Bozhko, V. S. Tiberkevich, and B. Hillebrands, Direct detection of magnon spin transport by the inverse spin Hall effect, Appl. Phys. Lett. 100, 082405 (2012).

[28] W. Han, S. Maekawa, and X.-C. Xie, Spin current as a probe of quantum materials, Nat. Mater. 19, 139 (2020).

[29] V. Cherepanov, I. Kolokolov, and V. L'vov, The saga of YIG: spectra, thermodynamics, interaction and relaxation of magnons in a complex magnet, Phys. Rep.-Rev. Sec. Phys. Lett. 229, 81 (1993).

[30] C. Dubs, O. Surzhenko, R. Linke, A. Danilewsky, U. Brückner, and J. Dellith, Sub-micrometer yttrium iron garnet LPE films with low ferromagnetic resonance losses, J. Phys. D 50, 204005 (2017).

[31] L. Mihalceanu, V. I. Vasyuchka, D. A. Bozhko, T. Langner, A. Y. Nechiporuk, V. F. Romanyuk, B. Hillebrands, and A. A.
Serga, Temperature-dependent relaxation of dipole-exchange magnons in yttrium iron garnet films, Phys. Rev. B 97, 214405 (2018).

[32] R. E. De Wames and T. Wolfram, Theory of surface spin waves in the Heisenberg ferromagnet, Phys. Rev. 185, 720 (1969).

[33] M. Mruczkiewicz, M. Krawczyk, G. Gubbiotti, S. Tacchi, Yu. A. Filimonov, D. V. Kalyabin, I. V. Lisenkov, and S. A. Nikitov, Nonreciprocity of spin waves in metallized magnonic crystal, New J. Phys. 15, 113023 (2013).

[34] A. G. Gurevich and G. A. Melkov, Magnetization Oscillations and Waves (CRC, New York, 1996).

[35] D. D. Stancil and A. Prabhakar, Spin Waves, Theory and Applications (Springer, Boston, 2009).

[36] A. Rückriegel, P. Kopietz, D. A. Bozhko, A. A. Serga, and B. Hillebrands, Magnetoelastic modes and lifetime of magnons in thin yttrium iron garnet films, Phys. Rev. B 89, 184413 (2014).

[37] B. Flebus, K. Shen, T. Kikkawa, K.-I. Uchida, Z. Qiu, E. Saitoh, R. A. Duine, and G. E. W. Bauer, Magnon-polaron transport in magnetic insulators, Phys. Rev. B 95, 144420 (2017).

[38] L. J. Cornelissen, K. Oyanagi, T. Kikkawa, Z. Qiu, T. Kuschel, G. E. W. Bauer, B. J. van Wees, and E. Saitoh, Nonlocal magnon-polaron transport in yttrium iron garnet, Phys. Rev. B 96, 104441 (2017)

[39] T. Kikkawa, K. Shen, B. Flebus, R. A. Duine, K.-I. Uchida, Z Qiu, G. E. W. Bauer, and E. Saitoh, Magnon Polarons in the Spin Seebeck Effect, Phys. Rev. Lett. 117, 207203 (2016).

[40] A. N. Slavin and Yu. K. Fetisov, Influence of the orientation of the static magnetic field on the dispersion curves for magnetization waves in yttrium iron garnet films, Sov. Phys.-Tech. Phys. 58, 1343 (1988).

[41] B. Hillebrands, Brillouin Light Scattering from Layered Magnetic Structure, in Light Scattering in Solids VII, edited by M. Cardona and G. Güntherodt (Springer-Verlag, Berlin, 2000).

[42] B. A. Kalinikos and A. N. Slavin, Influence of the orientation of the static magnetic field on the dispersion curves for magnetization waves in yttrium iron garnet films, J. Phys. C 19, 7013 (1986).

[43] K. Yu. Guslienko, S. O. Demokritov, B. Hillebrands, and A. N. Slavin, Effective dipolar boundary conditions for dynamic magnetization in thin magnetic stripes, Phys. Rev. B 66, 132402 (2002).

[44] K. Yu. Guslienko and A. N. Slavin, Boundary conditions for magnetization in magnetic nanoelements, Phys. Rev. B 72, 014463 (2005).

[45] R. V. Verba, A. Hierro-Rodriguez, D. Navas, J. Ding, X. M. Liu, A. O. Adeyeye, K. Y. Guslienko, and G. N. Kakazei, Spin-wave excitation modes in thick vortex-state circular ferromagnetic nanodots, Phys. Rev. B 93, 214437 (2016).

[46] G. N. Kakazei, G. R. Aranda, S. A. Bunyaev, V. O. Golub, E. V. Tartakovskaya, A. V. Chumak, A. A. Serga, B. Hillebrands, and K. Yu. Guslienko, Probing dynamical magnetization pinning in circular dots as a function of the external magnetic field orientation, Phys. Rev. B 86, 054419 (2012).

[47] B. W. Zingsem, M. Farle, R. L. Stamps, and R. E. Camley, Unusual nature of confined modes in a chiral system: Directional transport in standing waves, Phys. Rev. B 99, 214429 (2019).

[48] R. L. Stamps and B. Hillebrands, Dipole-exchange modes in multilayers with out-of-plane anisotropies, Phys. Rev. B 44, 5095 (1991). 
[49] See Supplemental Material at http://link.aps.org/supplemental/ 10.1103/PhysRevResearch.2.023324 for a movie showing a direct visualization of the time evolution of the mode profiles.

[50] N. Bloembergen, S. Shapiro, P. S. Pershan, and J. O. Artman, Cross-relaxation in spin systems, Phys. Rev. 114, 445 (1959).

[51] H. Kurebayashi, O. Dzyapko, V. E. Demidov, D. Fang, A. J. Ferguson, and S. O. Demokritov, Controlled enhancement of spin-current emission by three-magnon splitting, Nat. Mater. 10, 660 (2011).

[52] M. G. Cottam and A. N. Slavin, Fundamentals of linear and nonlinear spin-wave processes in bulk and finite magnetic samples, in Linear and Nonlinear Spin Waves in Magnetic Films and Superlattices, edited by M. G. Cottam (World Scientific, Singapore, 1994).

[53] S. M. Rezende and F. M. de Agnuiar, Spin-wave instabilities, auto-oscillations, and chaos in yttrium-iron-garnet, Proc. IEEE 78, 893 (1990).

[54] E. Schlömann, J. J. Green, and U. Milano, Recent developments in ferromagnetic resonance at high power levels, J. Appl. Phys. 31, S386 (1960).

[55] H. Suhl, The theory of ferromagnetic resonance at high signal powers, J. Phys. Chem. Solids 1, 209 (1957).

[56] A. Einstein and W. J. de Haas, Experimenteller nachweis der ampèreschen molekularströme, Verhandlungen. Dtsch. Phys. Gesellschaft. 17, 152 (1915).

[57] M. Matsuo, E. Saitoh, and S. Maekawa, Spin-mechatronics, J. Phys. Soc. Jpn. 86, 011011 (2017).

[58] S. Wintz, V. Tiberkevich, M. Weigand, J. Raabe, J. Lindner, A. Erbe, A. Slavin, and J. Fassbender, Magnetic vortex cores as tunable spin-wave emitters, Nat. Nanotechnol. 11, 948 (2016).

[59] Recently, Gunnink et al. proposed the same experiment and theoretically estimated the effect to be measurable, P. M. Gunnink, R. A. Duine, and A. Rückriegel, Electrical detection of uncon- ventional transverse spin-currents in obliquely magnetized thin films, arXiv:2003.12520.

[60] C. S. Davies and V. V. Kruglyak, Generation of propagating spin waves from edges of magnetic nanostructures pumped by uniform microwave magnetic field., IEEE Trans. Magn. 52, 2300504 (2016).

[61] G. Dieterle, J. Förster, H. Stoll, A. S. Semisalova, S. Finizio, A. Gangwar, M. Weigand, M. Noske, M. Fähnle, I. Bykova, J. Gräfe, D. A. Bozhko, H. Yu. Musiienko-Shmarova, V. Tiberkevich, A. N. Slavin, C. H. Back, J. Raabe, G. Schütz, and S. Wintz, Coherent Excitation of Heterosymmetric Spin Waves with Ultrashort Wavelengths, Phys. Rev. Lett. 122, 117202 (2019).

[62] C. W. Sandweg, M. B. Jungfleisch, V. I. Vasyuchka, A. A. Serga, P. Clausen, H. Schultheiss, B. Hillebrands, A. Kreisel, and P. Kopietz, Wide-range wavevector selectivity of magnon gases in Brillouin light scattering spectroscopy, Rev. Sci. Instrum. 81, 073902 (2010).

[63] S. O. Demokritov, B. Hillebrands, and A. N. Slavin, Brillouin light scattering studies of confined spin waves: linear and nonlinear confinement, Phys. Rep. 348, 441 (2001).

[64] S. M. Lindsay, M. W. Anderson, and J. R. Sandercock, Construction and alignment of a high performance multipass vernier tandem Fabry-Pérot interferometer, Rev. Sci. Instrum. 52, 1478 (1981).

[65] R. Mock, B. Hillebrands, and R. Sandercock, Construction and performance of a Brillouin scattering set-up using a triplepass tandem Fabry-Pérot interferometer, J. Phys. E 20, 656 (1987).

[66] B. Hillebrands, Progress in multipass tandem Fabry-Perot interferometry: I. A fully automated, easy to use, self-aligning spectrometer with increased stability and flexibility, Rev. Sci. Instrum. 70, 1589 (1999). 